\title{
Third-Based Facial Similarities and Differences of Monozygotic Twins: A Stereophotogrammetric 3D Assessment
}

\author{
Valentina PUCCIARELLI ${ }^{1}$, Daniele GIBELLI ${ }^{1}$, Danilo DE ANGELIS ${ }^{2}$, Pasquale POPPA ${ }^{2}$, \\ Marco CUMMAUDO ${ }^{2}$, Marina CODARI ${ }^{3}$, Claudia DOLCl ${ }^{1}$, Chiarella SFORZA ${ }^{1^{*}}$ \\ ${ }^{1}$ Dipartimento di Scienze Biomediche per la Salute, Università degli Studi di Milano, Milano, Italy; \\ ${ }^{2}$ LABANOF - Laboratorio di Antropologia e Odontologia Forense, Sezione di Medicina Legale, \\ Dipartimento di Scienze Biomediche per la Salute, Università degli Studi di Milano, Milan, Italy; \\ ${ }^{3}$ Unit of Radiology, IRCCS Policlinico San Donato, San Donato Milanese, Milan, Italy
}

DOI: 10.15221/17.226 http://dx.doi.org/10.15221/17.226

\begin{abstract}
The genetic background of the craniofacial development is a sensitive topic: in this context twin studies have had a relevant role as they allow to understand the effect of genes on the anatomical variability observed in the population. In particular, monozygotic twins (MZT) share the same DNA and allow to evaluate the genetic component of a specific morphology. In order to understand the similarities and differences on the facial morphology of MZT in different portions of the face, a thirdbased, superimposition approach was applied to the 3D facial scans of 10 couples of MZT. The experimental subjects were acquired through a stereophotogrammetric system, after the identification of a set of reference landmarks on their facial surfaces. The landmarks were used to segment facial areas of interest from the $3 \mathrm{D}$ reconstructions and to subdivide them into thirds, according to the territories of distribution of trigeminal branches for somatic sensitivity. The left and right upper, middle and lower facial thirds of each MZT couple were pairwise superimposed and the root mean square (RMS) point-to-point distances were automatically calculated. Data were statistically analysed through a two-way ANOVA, setting the level of significance at $5 \%$. Post-hoc tests were performed with the necessary reductions in the degrees of freedom. Results revealed statistically significant differences among thirds $(p<0.05)$, while no differences were found for facial sides $(p>0.05)$ or for the third $x$ side interaction $(p>0.05)$. Post hoc tests showed statistically significant differences between the upper and the lower facial thirds, and the middle and lower facial thirds $(p<0.05)$, with the middle and lower thirds being the more different between the couples. In conclusion, stereophotogrammetric techniques can be valid instruments to analyse the facial morphology of MZT. The acquisition procedure is easy to perform, fast and free from risk, being suitable to obtain multiple subsequent 3D reconstructions. These reconstructions can be superimposed and locally analysed in order to provide an anatomically-based, detailed description of the most similar and different facial areas, useful for the evaluation of the genetic components of a specific morphology.
\end{abstract}

Keywords: Monozygotic twins, stereophotogrammetry, face, facial thirds

\section{Introduction}

The genetic background of the craniofacial morphology is a complex topic: epigenetic, gene dosage and craniofacial enhancers may modify its development, thus hindering its clarification [1-3]. However, in this context twin studies may play a significant role: monozygotic twins (MZT) share the same DNA and are appropriate experimental models to understand the genetic and environmental components of a specific morphology [1].

Previous studies have already investigated twin facial morphology, using both two and threedimensional (3D) imaging systems. Nevertheless, the advancement of the technologies in the last years has enhanced the number of 3D studies in this field [4-7].

Among the 3D acquisition systems, optical instruments such as laser scanners and stereophotogrammetry have permitted to reproduce 3D facial morphology safely and rapidly, in order to perform reliable morphometric analyses of the face $[8,9]$.

Their valuable advantage is the non invasiveness, which allows to perform multiple facial acquisitions in healthy subjects that have no medical reasons to undergo other invasive or fastidious 3D imaging procedures [10].

\footnotetext{
* *chiarella.sforza@unimi.it; +39 02503 15387; www.scibis.unimi.it
} 
Moreover, 3D models obtained from optical instruments allow to perform not only linear and angular measurements, but also superimpositions [11]. The registration of the acquired models consents to calculate point-to point distances between them (Root Mean Square, RMS), which provide a quantitative index of their differences and similarities.

At this purpose, a previous study executed by Djorjevic et al. investigated the symmetry of the facial thirds of a sample of $19 \mathrm{MZT}$ and 18 dizygotic twins imaged through a laser scanner and analysed through superimpositions [12]. The protocol required a set of two separate facial scans (right and left sides of the face), that were acquired one after the other but with some possible modifications of facial mimicry (eyes, nose and mouth regions). In contrast, stereophotogrammetric instruments have a shorter acquisition time, and need a single acquisition, thus limiting possible bias due to involuntary movements during the different phases of scan.

A part from that previous study [12], performed through laser scanner, to the best of our knowledge no study has so far applied stereophotogrammetric 3D-3D superimposition to the analysis of facial morphology in twins.

The aim of the current investigation is to evaluate differences and similarities on the superimposed facial thirds of couples of MZT acquired through stereophotogrammetry. An anatomic criterion, previously validated by our group, is used in the definition of the thirds [13]. Since the common genetic background of the MZT, the information obtained in this study can be of interest in assessing the respective influences by genes or environment on facial morphology.

\section{Methods}

\subsection{Involved subjects}

For this study 6 couples of male and 4 couples of female MZT, aged between 25 and 69 years, were recruited. All the experimental subjects were Caucasoid and without a previous history of facial surgery or trauma. Zygosity was ascertained by genetic tests, previously performed by the subjects for reasons not related with the study.

All the procedures were not dangerous or invasive and were performed according to the Declaration of Helsinki and after all the involved subjects had read, understood and voluntarily signed an informed consent, according to local and international ethical rules.

\subsection{Image acquisition}

All the subjects were acquired with the stereophotogrammetric system VECTRA-3D® M3 (Canfield Scientific, Inc., Fairfield, NJ).

VECTRA is an optical instrument, with a geometry resolution of $1.2 \mathrm{~mm}$, that allows to obtain threedimensional reconstructions of the face [14]. The twins were imaged following a standardized procedure: they sat in front of the system, with closed mouth and teeth in loose contact. Furthermore, they were asked to keep a neutral facial expression $[15,16]$.

Before the facial acquisitions, 50 anthropometric landmarks were marked on their faces. Collocation of facial landmarks was performed by a trained and expert operator, using a black liquid eyeliner for makeup. For all the acquisitions, a standardized protocol defined in the laboratory and already used in previous studies was followed. A detailed description of the set of cutaneous landmarks can be found elsewhere [17].

\subsection{Identification of Facial Area of Interest (FAl) and facial thirds segmentation}

The images were processed using Mirror ${ }^{\circledR}$ (Canfield Scientific Inc., Fairfield, NJ), the software of the stereophotogrammetric system. The first step consisted in the selection of a facial area of interest (FAI) to be analyzed for each subject.

This surface was semi-automatically detected: once the external-most facial landmarks had been manually selected, the software was able to select the facial inner area and to remove the external regions, such as hair and neck. Ears were excluded too (Figure 1). The procedure is described in detail in a previous study performed by our group, and it was found to be repeatable [13].

For all the twin couples, the FAls of the corresponding siblings were registered. Registration was performed superimposing, conventionally, the face of the second-born twin on that of the first. It was automatically done by the stereophotogrammetric system software, which registered the faces according to the least differences between corresponding points on them. 
Considering the registered FAls of all the couples of MZT, they were subdivided in hemifaces and each facial half was divided in upper, middle and lower facial thirds. The segmentation was performed following the territories of distribution of the trigeminal branches for somatic sensitivity, according to a segmentation protocol defined by Codari et al. (Figure 2) [13].

RMS distances for upper, middle and lower facial thirds, for both the left and right sides of the face, were automatically calculated by the software. Possible differences due to the side in different thirds were assessed as well.

A color-coded map, resulting from the facial thirds superimpositions, was automatically generated by the software. Figure 3 shows an example of map, obtained for the lower third in a couple of MZT. Similar areas between the couples of MZT were represented in green, while differences were depicted in red and blue. In particular, red areas indicate negative differences, while blue indicate positive deviations.

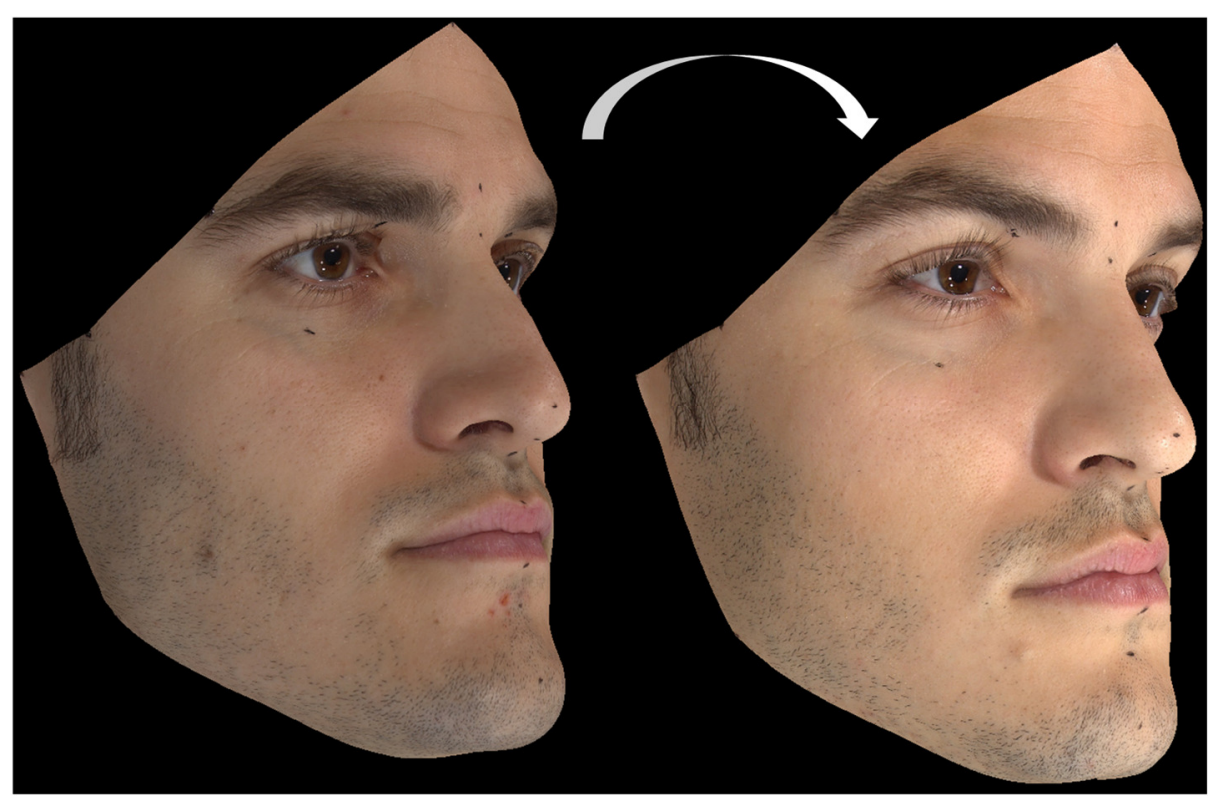

Fig. 1. Semiautomatically segmented FAls of a couple of MZT before facial registration.

The white arrow indicates the superimposition procedure performed, as convention, registering the face of the second-born twin on that on the first .

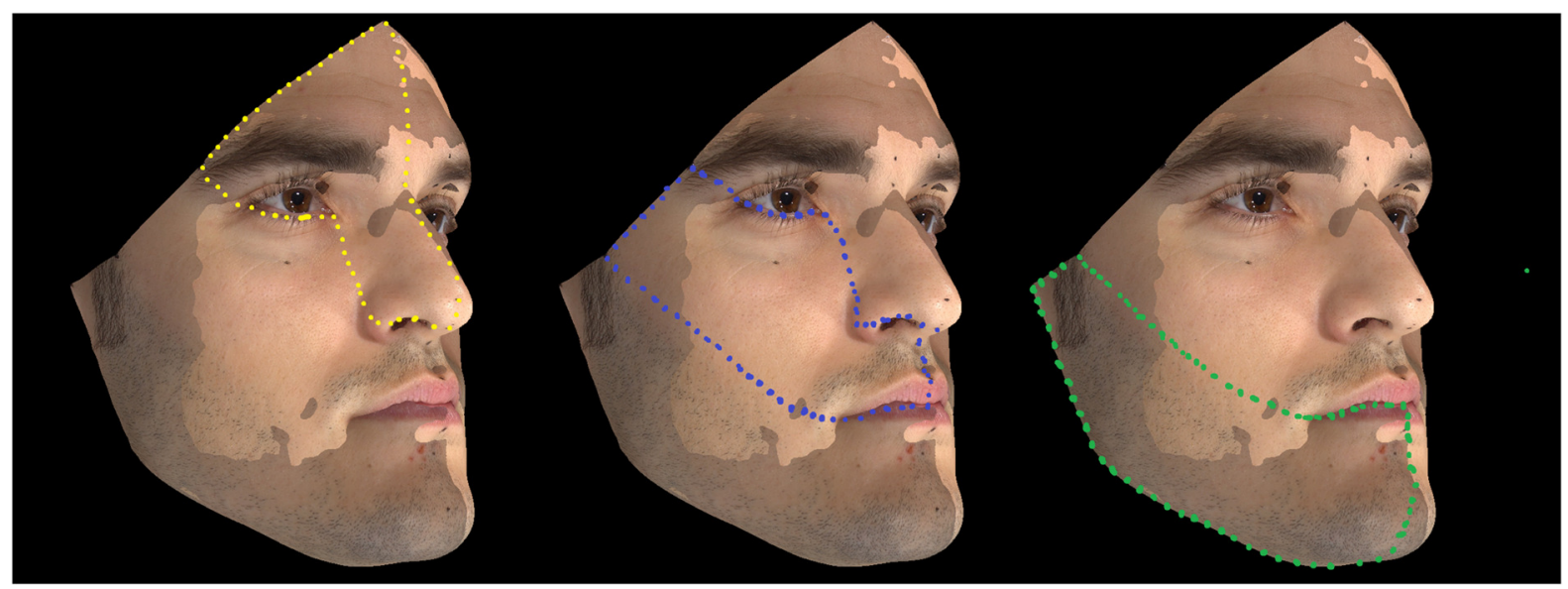

Fig. 2. Upper (yellow), middle (blue) and lower (green) facial thirds, segmented on the registered FAls of a couple of MZT. Segmentation was performed according to the territories of distribution of the trigeminal branches for somatic sensitivity. 


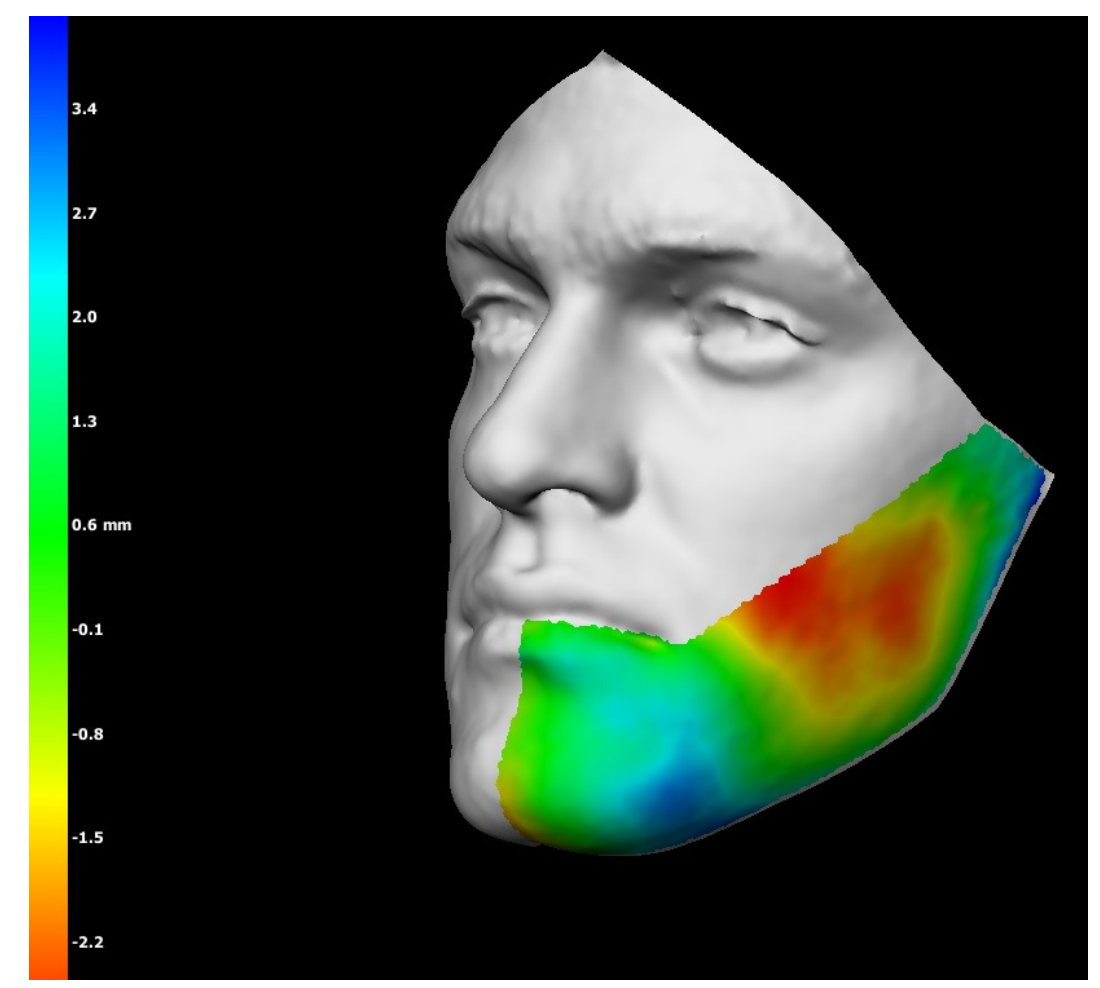

Fig. 3. Colour coded map showing areas of the lower facial third that are similar (green) or differ (red and blue) in a couple of MZT.

\section{Statistical analysis}

Normality of all data was checked using Jarque Bera test. A two-way ANOVA was executed on RMS values from facial thirds superimposition on corresponding twins (Factor 1: facial third; Factor 2: side; the facial third $x$ side interaction was also computed). The level of significance was set at $5 \%$. Posthoc tests were performed with the necessary reductions in the degrees of freedom.

\section{Results}

Table 1 reports the RMS values calculated from facial thirds superimpositions on couples of corresponding twins, while table 2 shows the results for the two-way ANOVA performed on the previously calculated RMS values. The results of post-hoc tests are shown too. Statistically significant differences were found according to thirds $(p<0.05)$, not according to side $(p>0.05)$. Post-hoc test revealed that differences between the lower facial thirds and the other two portions were statistically significant $(p<0.05)$. Overall, the lower facial third was significantly more discordant between MZT than the other parts of the face.

Table 1. The RMS values (mean $\pm S D$ ) calculated from facial thirds superimpositions on couples of corresponding twins

\section{RMS [mm]}

\begin{tabular}{ccccc}
\hline & & Upper third & Middle third & Lower third \\
\hline $\begin{array}{c}\text { left facial } \\
\text { side }\end{array}$ & Mean & 1.2 & & \\
& SD & 0.3 & 0.5 & 1.7 \\
$\begin{array}{c}\text { right facial } \\
\text { side }\end{array}$ & Mean & 1.1 & 1.2 & 0.7 \\
& SD & 0.2 & 0.4 & 1.8 \\
& & & & 0.5 \\
\hline
\end{tabular}


Table 2. Two-way ANOVA and post hoc tests of RMS values from facial thirds superimposition on corresponding twins.

\begin{tabular}{ll}
\hline Two- way ANOVA & \\
\hline facial thirds & $\mathrm{p}=0.0004$ \\
facial side & $\mathrm{p}=1$ \\
facial thirds $x$ side & $\mathrm{p}=0.81$ \\
& \\
\hline Post hoc tests & $\mathrm{p}=0.39$ \\
upper third vs middle third & $\mathrm{p}=0.002$ \\
upper third vs lower third & $\mathrm{p}=0.006$ \\
middle third vs lower third & \\
\hline
\end{tabular}

\section{Discussion}

Despite the role of molecular biology in twin studies is supposed to be predominant, morphological investigations have given a significant contribution to this field, due to technologic advancement [17]. The current study represents a morphological stereophotogrammetric evaluation of the similarities and differences of the facial thirds of 10 couples of MZT, through a superimposition approach.

A similar approach was applied by a previous study, which investigated the symmetry of the facial thirds of a sample of mono- and dyzigotic twins [12]. In that study, the acquisitions were performed using a laser scanner, and in particular a device that required a separate capture of the right and left facial sides and their successive automatical combination into a single image.

On the contrary, the stereophotogrammetric instrument used in the current study allows the complete facial acquisition in a very short time and with a single scan [14]. This aspect has an important role since facial involuntary movements can affect the 3D reconstruction and the rapidity of the acquisition system must be considered, when performing morphological evaluations [18].

Furthermore, our study considers an anatomic subdivision of the face in thirds, that follows the territories of distribution of the main trigeminal branches for somatic sensitivity. Nevertheless, in other studies, the subdivision of the face in thirds was performed, arbitrarily, through horizontal planes $[19,20]$.

Since the aim of this investigation was the assessment of similarities and differences in facial areas of couples of individuals who share the same DNA, an anatomic detailed assessment seemed to be mandatory, considering the future possibility to correlate the genetic background of the twins to the morphological features.

Our results show the presence of statistically significant differences among the MZT facial thirds, while no differences are found for the facial side and the thirds $x$ side interaction. In accordance with existing literature, most of the differences involve the lower facial thirds, thus indicating a different influence of the genetic factors in different facial areas [12,20]. Interestingly, although the analysis was performed through another method of 3D image acquisition, the present results confirm the study by Djordjevic et al. based on laser scanner technology [3]: this concordance strengthened once more the importance of environment in determining the lower third which proved to be the most different part of the face in twins.

Further investigations on a larger sample are mandatory to clarify genetic and environmental relationships on MZT. Stereophotogrammetric techniques can be considered valid tools for these types of investigations.

\section{References}

[1] J. Roosenboom et al. "Exploring the Underlying Genetics of Craniofacial Morphology through Various Sources of Knowledge" Biomed Res Int, Vol. 2016, 2016, pp.1-9, http://dx.doi.org/10.1155/2016/3054578.

[2] C. Attanasio et al. "Fine Tuning of Craniofacial Morphology by Distant-Acting Developmental Enhancers", Science, Vol. 342, No. 6157, 2013, pp. 1-20, http://dx.doi.org/ 10.1126/science.1241006. 
[3] J. Djordjevic et al. "Genetic and Environmental Contributions to Facial Morphological Variation: A 3D Population-Based Twin Study", PLoS One, Vol. 11, No. 9, 2016, pp.1-20, http://dx.doi.org/1010.1371/journal.pone.0162250.

[4] LA Kohn. "The role of genetics in craniofacial morphology and growth", Annu Rev Anthr, Vol. 20, 1991, pp. 161-78.

[5] PH. Burke, MJ. Healy " A serial study of normal facial asymmetry in monozygotic twins", Ann Hum Biol, Vol. 20, No. 6, 1993, pp. 527-34.

[6] JP. Moss. "The use of three-dimensional imaging in orthodontics", Eur J Orthod, Vol. 28, 2006, pp. 416-25.

[7] FB. Naini, JP. Moss. "Three-dimensional assessment of the relative contribution of genetics and environment to various facial parameters with the twin method", Am J Orthod Dentofacial Orthop, Vol. 126, No. 6, 2004, pp. 655-65.

[8] V. Pucciarelli et al. "The Face of Glut1-DS Patients: A 3D Craniofacial Morphometric Analysis", Clin Anat, Vol. 30, 2017, pp. 644-652, http://dx.doi.org/10.1002/ca.22890.

[9] C. Dolci et al. "3D Morphometric Evaluation of Craniofacial Features in Adult Subjects with Marfan Syndrome", Proc 7th Int Conf 3D Body Scanning Technol Lugano, Switzerland, 30Nov1 Dec 2016; pp. 98-104, http://dx.doi.org/10.15221/16.098.

[10] C. Sforza et al. "Soft- and hard-tissue facial anthropometry in three dimensions: what's new. J Anthropol Sci, Vol. 91, 2013, pp. 159-84, http://dx.doi.org/10.4436/jass.91007.

[11] V. Pucciarelli et al. "Stereophotogrammetric Evaluation of Labial Symmetry After Surgical Treatment of a Lymphatic Malformation" J Craniofac Surg, Vol. 28, No. 4, 2017, pp. e355-e8, http://dx.doi.org/ 10.1097/SCS.0000000000003601.

[12] J. Djordjevic et al. "Three-dimensional analysis of facial shape and symmetry in twins using laser surface scanning", Orthod Craniofac Res, Vol. 16, No. 3, 2013, pp. 146-60, http://dx.doi.org/10.1111/ocr.12012.

[13] M. Codari et al. "Facial thirds e based evaluation of facial asymmetry using stereophotogrammetric devices: Application to facial palsy subjects", J Cranio-Maxillofacial Surg, Vol. 45, No. 1, 2017, pp. 76-81, http://dx.doi.org/10.1016/j.jcms.2016.11.003

[14] http://www.canfieldsci.com/imaging-systems/vectra-m3-3d-imaging-system/accessed on June 27, 2017.

[15] V. Pucciarelli et al. "Three-Dimensional Craniofacial Features of Glut1 Deficiency Syndrome Patients", Proc 6th Int Conf 3D Body Scanning Technol Lugano, Switzerland, 27-28 Oct 2015, http://dx.doi.org/10.15221/15.061.

[16] C. Dolci et al. "3D Craniofacial Morphometric Analysis of Young Subjects with Marfan Syndrome: A Preliminary Report" Proc 6th Int Conf 3D Body Scanning Technol Lugano, Switzerland, 27-28 Oct 2015, http://dx.doi.org/10.15221/15.054.

[17] TE. Hughes et al. "The teeth and faces of twins: providing insights into dentofacial development and oral health for practising oral health professionals" Aust Dent J, Vol. 59, Suppl 1, 2014, pp. 101-16, http://dx.doi.org/10.1111/adj.12101.

[18] GA. Ramieri et al. "Reconstruction of facial morphology from laser scanned data. Part I: reliability of the technique", Dentomaxillofac Radiol, Vol. 35, No. 3, 2006, pp. 158-64, http://dx.doi.org/10.1259/dmfr/43516583.

[19] HO. Taylor et al. "Quantitative facial asymmetry: using three-dimensional photogrammetry to measure baseline facial surface symmetry", J Craniofac Surg, Vol. 25, No. 1, 2014, pp. 124288, http://dx.doi.org/10.1097/SCS.0b013e3182a2e99d

[20] U. Ozsoy. "Comparison of Different Calculation Methods Used to Analyze Facial Soft Tissue Asymmetry: Global and Partial 3-Dimensional Quantitative Evaluation of Healthy Subjects", J Oral Maxillofac Surg, Vol. 74, No. 9, 2016, pp. 1847.e1-9, http://dx.doi.org/10.1016/j.joms.2016.05.012. 